\title{
HER2 immunohistochemical and fluorescence in situ hybridization discordances in invasive breast carcinoma with micropapillary features
}

\author{
Rachel L Stewart ${ }^{1}$, Justin E Caron ${ }^{1}$, Evin H Gulbahce ${ }^{1}$, Rachel E Factor ${ }^{1}$, \\ Katherine B Geiersbach ${ }^{2}$ and Erinn Downs-Kelly ${ }^{3}$ \\ ${ }^{1}$ Department of Pathology, ARUP Laboratories, University of Utah Medical Center, University of Utah, Salt \\ Lake City, UT, USA; ${ }^{2}$ Department of Laboratory Medicine and Pathology, Mayo Clinic, Rochester, MN, USA \\ and ${ }^{3}$ Robert J. Tomsich Pathology and Laboratory Medicine Institute, Cleveland Clinic, Cleveland, OH, USA
}

\begin{abstract}
The 2013 American Society of Clinical Oncology/College of American Pathologists (ASCO/CAP) recommendations for HER2 testing contain a recommendation for pathologists with respect to invasive micropapillary carcinoma. The guidelines suggest that HER2 immunohistochemical staining that is intense but incomplete and would be considered 1+ may actually be HER2-amplified by fluorescence in situ hybridization. Thus, pathologists should consider reporting the immunohistochemistry as equivocal (2+) and employ an alternative testing methodology. This recommendation is based largely on one paper wherein the authors tested a series of 22 micropapillary carcinomas that were considered $1+$ by immunohistochemistry and identified HER2 amplification in one case $(5 \%)$. In order to assess for a possible discordance between HER2 immunohistochemistry and fluorescence in situ hybridization, we evaluated a series of invasive carcinomas with micropapillary features using both methodologies. As described by the WHO, invasive carcinomas with micropapillary features have small, hollow, or morula-like clusters of cells surrounded by clear stromal spaces. All cases had HER2 immunohistochemistry and fluorescence in situ hybridization performed, and for cases with equivocal fluorescence in situ hybridization results, an alternative Chromosome 17 probe (RA/1) was employed. All assays were scored according to the 2013 ASCO/CAP guidelines. Specifically for this study, immunohistochemistry was scored irrespective of the presence of micropapillary features. Overall, we identified HER2 amplification in $21(47 \%)$ of the cases assayed, with the corresponding immunohistochemistry being $1+(n=9), 2+(n=11)$, and $3+(n=1)$. The ASCO/CAP recommendation that this morphology may deviate from the typical staining pattern is highlighted, as we found that $43 \%$ of cases with micropapillary features and HER2 staining that would otherwise be scored as 1+ were HER2-amplified by fluorescence in situ hybridization. This study supports the ASCO/CAP recommendation that pathologists should consider reporting immunohistochemistry in this morphology as equivocal and perform reflex testing using in situ hybridization.
\end{abstract}

Modern Pathology (2017) 30, 1561-1566; doi:10.1038/modpathol.2017.65; published online 28 July 2017

HER2 (ERBB2) expression in breast carcinoma is an established predictive biomarker for response to anti-HER2 therapies. As such, laboratories are charged with the accurate assessment of HER2 by either of two FDA-approved testing modalities, which include immunohistochemistry and in situ hybridization. The American Society of Clinical

Correspondence: Dr Rachel L Stewart, DO, PhD, Department of Pathology, ARUP Laboratories, University of Utah Medical Center, University of Utah, 500 Chipeta Way, MS-115, Salt Lake City, UT 84108-1221, USA.

E-mail: rachel.stewart@aruplab.com

Received 19 January 2017; revised 3 May 2017; accepted 6 May 2017; published online 28 July 2017
Oncology and the College of American Pathologists (ASCO and CAP) have provided guidelines that aim to improve concordance between laboratories and that attempt to minimize pre-analytical, analytical, and post-analytical variabilities. ${ }^{1}$ Each iteration of the ASCO/CAP guidelines is an evolution that attempts to ensure that HER2 testing correctly identifies patients who may derive a benefit from HER2-targeted therapy while avoiding the possibility of false-positives.

Tumor morphology has an important role in the updated ASCO/CAP guidelines, with recommendations included for situations in which the histopathologic features may suggest a possible discordance in HER2 testing. ${ }^{1}$ For example, an alternate HER2 test 
should be considered when Grade 1 tumors have an initial HER2-positive result. Similarly, if an initial HER2 test on a core needle biopsy is negative, a new HER2 test should be considered on the excision if the tumor is Grade 3. The ASCO/CAP 2013 guidelines suggest that micropapillary carcinoma with HER2 immunohistochemistry staining that is intense but incomplete (basolateral or U-shaped) and would be considered 1+ may actually be HER2-amplified by fluorescence in situ hybridization. Thus, it is recommended that pathologists consider reporting these specimens as equivocal (2+) and perform an alternative testing methodology. This recommendation is based largely on a study by Vingiani et $a l^{2}{ }^{2}$ wherein the authors tested a series of 22 micropapillary carcinomas that were considered $1+$ by HercepTest and subsequently identified HER2 amplification in $5 \%$ of cases. We sought to evaluate this possible discordance between immunohistochemistry and fluorescence in situ hybridization in a series of invasive carcinomas with micropapillary features that had both testing methodologies performed.

\section{Materials and methods}

After obtaining Institutional Review Board approval, invasive carcinomas with micropapillary features were identified over a 9-month time frame and were culled from routine sign out of HER2 tests in a reference laboratory. A series of 45 cases of invasive carcinoma with micropapillary features was identified, with all cases having dual test results for both HER2 immunohistochemistry and fluorescence in situ hybridization. Primary HER2 testing was the reason for submission to the lab in $47 \%(21 / 45)$ of cases with an additional $13 \%(6 / 45)$ submitted for second opinion testing, or dual testing for results of 0 or $3+$. The remaining 18 cases $(40 \%)$ were submitted for reflex HER2 fluorescence in situ hybridization following an equivocal immunohistochemistry result from the originating facility. Our laboratory performs HER2 immunohistochemistry using the DAKO HercepTest and HER2 fluorescence in situ hybridization using the HER2 IQFISH pharmDx test (Carpinteria, CA); all tests were performed according to the manufacturer's instructions and all fluorescence in situ hybridization cases were scored according to the 2013 ASCO/CAP guidelines. Equivocal fluorescence in situ hybridization cases were reflexed to a laboratory-developed assay as our group has previously described, ${ }^{3}$ which utilizes a dual probe set (Agilent, Santa Clara, CA) targeting RAI1 as a different control locus on the short arm of chromosome 17 as well as a HER2 probe. The results of the reflex fluorescence in situ hybridization assay are interpreted using the HER2/RAI1 ratio ( $\geq 2$ is amplified, $<2$ is non-amplified). A subset of the cases included in this study was referred for HER2 fluorescence in situ hybridization testing because of either equivocal immunohistochemistry results or client preference that fluorescence in situ hybridization be the primary testing methodology. For cases that were received for fluorescence in situ hybridization testing, a corresponding HercepTest was performed on residual slides solely on a research basis. The remaining cases were identified from client cases submitted for HercepTest. The clinically reported HercepTests were scored according to the 2013 ASCO/CAP guidelines and adherence to the recommendation for those with micropapillary features was followed. In reporting these cases, the micropapillary features were denoted in a comment, the material was scored as equivocal, and reflex testing with HER2 fluorescence in situ hybridization was performed. Specifically for this study, the HercepTest material was reviewed and scored by two breast pathologists (EG and EDK) who were blinded to the fluorescence in situ hybridization results. The ASCO/CAP key recommendation relating to this specific morphology was exempted from this scoring process, and cases were scored irrespective of morphology using the guidelines as follows: a positive (3+) HER2 test requires that $>10 \%$ of invasive tumor cells display strong and circumferential membrane staining, whereas a negative (0) HER2 test is defined as no staining observed or membrane staining that is incomplete, faint/barely perceptible, and within $\leq 10 \%$ of the invasive tumor cells. Cases are scored as (1+) if there is incomplete membrane staining that is faint/barely perceptible and within $>10 \%$ of invasive tumor cells, whereas equivocal $(2+)$ cases include those with weak to moderate complete membrane staining in $>10 \%$ of invasive tumor cells. ${ }^{1,4}$

\section{Results}

A total of 45 cases with micropapillary features were identified, representing 29 core needle biopsies and 16 resection specimens (one representative slide each). The percentage of micropapillary features present ranged from 30 to $100 \%$, with tumors being either Nottingham Grade 2 or Grade $3(n=13$ and $n=32$, respectively). The FDA-approved probe set (HER2 IQFISH pharmDx) identified $18 / 45$ (40\%) as HER2-non-amplified, whereas the corresponding HercepTest for this group included scores of 0 $(n=3), 1+(n=8)$, and $2+(n=7)$. The FDA-approved probe set identified 14/45 (31\%) as HER2-amplified ( $n=4$ by copy number $\geq 6$ with copy numbers of 6.0 , $6.5,6.5$, and $7.3 ; n=10$ by ratio with HER2/CEN-17 ratios of 2.1 to $>2.6$ ), whereas the corresponding HercepTest results for this group included scores of $1+(n=7), 2+(n=6)$, and $3+(n=1)$. The FDAapproved probe set identified 13/45 (29\%) as HER2 equivocal with reflex testing to the alternative probe set. This alternate probe set identified five of these cases as non-amplified with corresponding HercepTest results of $1+(n=1)$ and $2+(n=4)$. The reflex probe test identified seven cases as amplified 
(HER2/RAI1 ratio ranging from 2.0 to 2.5 ) with corresponding HercepTest results of $1+(n=2)$ and $2+(n=5)$. One case could not be resolved with the reflex probe set owing to an apparent deletion of the RAI1 probe (RAI1 copy number of 1.2). Overall, we identified HER2 amplification in 21/45 (47\%) of the cases assayed, with the corresponding immunohistochemistry being $1+(n=9), 2+(n=11)$, and $3+$ $(n=1)$.

With respect to HER2 immunohistochemistry in the HER2-amplified subset, only one of the HER2-amplified tumors had a 3+ corresponding HercepTest; all other HER2-amplified tumors had either weak or only moderate intensity staining. Of the 11 amplified cases that were scored as $2+$, staining was non-uniform, weak to moderate, and deposited along the cell membrane at the apical surface, the lateral surface, or at the periphery of the morula. The nine fluorescence in situ hybridization-amplified cases that had a corresponding 1+ HercepTest are detailed in Table 1 and Figure 1. With respect to tumor morphology, the cases that were not $100 \%$ micropapillary had a component of invasive ductal carcinoma, NOS. The HercepTest score within these different morphologies was similar, meaning that no particular component was of a higher immunohistochemistry score.

\section{Discussion}

We found that HER2 protein localization in invasive carcinoma with micropapillary features deviates from the typical circumferential pattern that is often seen in invasive breast carcinomas of no special type. This phenomenon has been appreciated by others, with the first report of this finding from Vingiani et al, who identified one case of HER2 amplification in a series of 22 that were $1+$ by immunohistochemistry. ${ }^{2}$ In a recent study evaluating HER2 expression in 52 cases of micropapillary carcinoma, Yang et al found that, in a number of HER2-amplified cases, although HER2 protein expression was detected at the cell-cell and basolateral membranes, it was not detected along the cytoplasmic membrane facing the stroma. ${ }^{5}$ In their series, they found that of those cases that were $1+$ by immunohistochemistry $(n=25)$, one was HER2amplified and an additional two cases were equivocal by fluorescence in situ hybridization. Notably, all of the cases in their study that had 2+ HER2 expression by immunohistochemistry $(n=12)$ were amplified by fluorescence in situ hybridization. These studies suggest that it may be possible to miss patients who are indeed HER2-positive if using immunohistochemistry and not having an awareness of the altered immunoreactivity associated with this morphology.

Incomplete membrane staining has been recognized as an important characteristic in gastric carcinoma where HER2 expression tends to be basolateral rather than circumferential. ${ }^{6}$ For this reason, HER2 positivity (3+) in gastric cancer has been defined as 'strong complete, basolateral, or lateral membranous reactivity in $\geq 10 \%$ of tumor cells' for the purpose of some clinical trials. ${ }^{7}$ As this pattern of incomplete expression is also present in some cases of micropapillary carcinoma associated with HER2 amplification, our study provides additional support for the ASCO/CAP recommendation that an alternate testing methodology be considered in cases of micropapillary carcinoma with intense but incomplete expression of HER2. ${ }^{1}$ Furthermore, the current study suggests that immunoreactivity may even be weak in cases that are HER2-amplified.

In micropapillary carcinomas, cell clusters are often separated from the extracellular matrix by a clear clefted space in which the apical surface of the cluster is oriented toward the basement membrane. This peculiar architecture is thought to be due in part to altered expression and subcellular localization of proteins controlling cell polarity, as well as to mutations in the genes encoding these proteins. ${ }^{8}$ Notably, mutations in genes involved in cell polarity (FMN2), ciliogenesis (DNAH9), and cytoskeletal organization (UBR4, PTPN21) have been identified in micropapillary carcinomas, although at a relatively low frequency. ${ }^{8}$ LIN7A has been identified as a gene that is upregulated in micropapillary carcinomas and that may be responsible for altered apicobasal polarity in these neoplasms. ${ }^{9}$ Interestingly, LIN7A appears to be required for HER2 localization and maintenance at the basolateral surface of cells, and has also been implicated in promoting a proliferative and invasive phenotype in cell lines and animal models.9,10

Previous studies have shown that a subset of micropapillary carcinomas tend to cluster as Luminal B using gene expression profiling, ${ }^{8,11}$ and this molecular subtype can demonstrate HER2 gene amplification as well as high expression of genes related to proliferation. ${ }^{11,12}$ HER2 protein expression by immunohistochemistry has been observed in micropapillary carcinomas and ranges from 12.5 to $95 \%$ of cases in the studies reviewed..$^{2,13-17}$ This wide range of reported HER2 expression in micropapillary carcinomas may partly be due to variability in HER2 scoring, as a subset of these studies was performed before either the 2007 or 2013 ASCO/CAP guidelines were in place.

There is undoubtedly bias in our study population as the material is largely from a reference laboratory and this material is enriched for equivocal cases that needed reflex fluorescence in situ hybridization or an alternative method of testing. However, this series highlights a not uncommon discordance between immunohistochemistry and fluorescence in situ hybridization in the setting of invasive carcinomas with micropapillary features. The current study and studies by others have found that a subset of micropapillary carcinomas with HER2 amplification have incomplete membranous staining that would be scored as $1+$ or $2+$ by immunohistochemistry. ${ }^{2,5}$ To put these numbers into perspective with respect to 


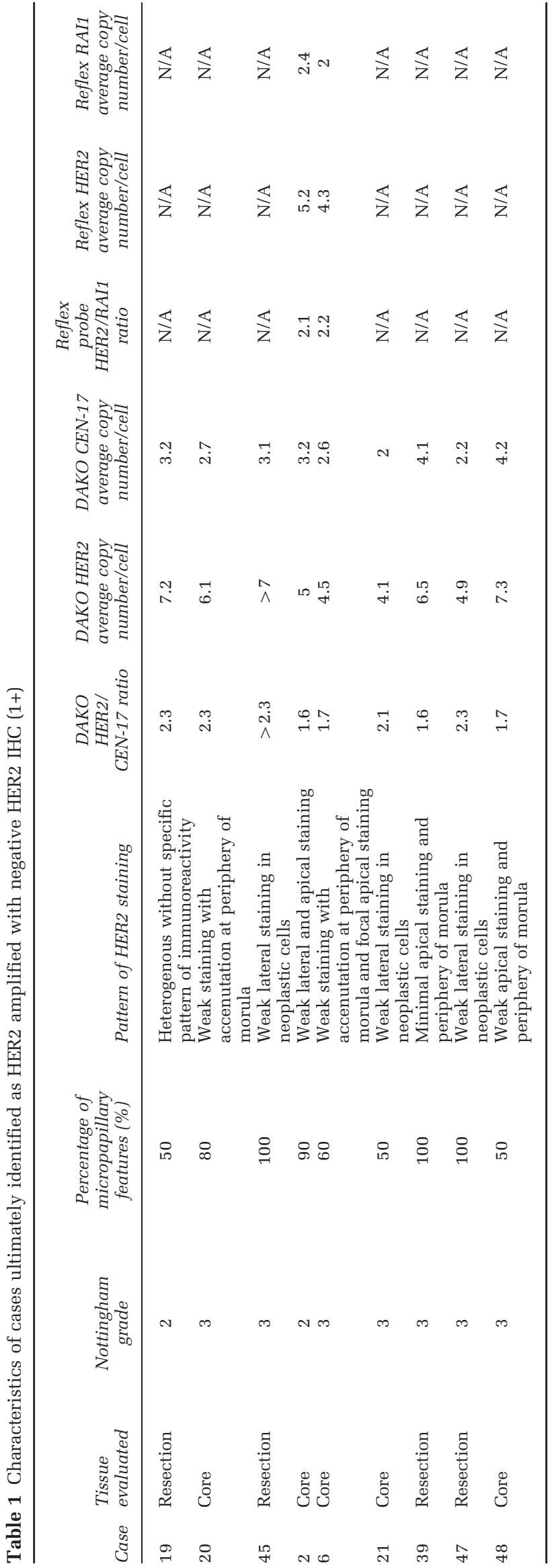

HER2 testing in our laboratory, the statistics for a 1-year period using the 2013 ASCO/CAP guidelines demonstrated the following: an FDA-approved dual probe set (cases solely tested with fluorescence in situ hybridization) identified 252/1242 (20.4\%) of cases as HER2-amplified, 92/1242 (7.4\%) as equivocal, $886 / 1242(71.3 \%)$ as HER2-non-amplified, and $12 / 1242(0.9 \%)$ as indeterminate. These rates of amplified, equivocal, and non-amplified are similar to those published by other reference laboratories and are similar to rates obtained in clinical trials with centralized testing. During this same time course, stand-alone immunohistochemistry testing identified $195 / 1684(11.6 \%)$ as positive $(3+), 495 / 1684(29.4 \%)$ as equivocal $(2+)$, and $994 / 1684(59 \%)$ as negative (0 to $1+$ ). During that time, a single case was scored as indeterminate for HER2 immunohistochemistry. Dual testing (both immunohistochemistry and fluorescence in situ hybridization) was performed in 411 cases. Of those cases with dual testing, 20/411 (4.9\%) were scored as (0 to $1+$ ), with the corresponding fluorescence in situ hybridization identifying $3 / 20(15 \%)$ as HER2amplified, $1 / 20(5 \%)$ as fluorescence in situ hybridization equivocal, and 16/20 (80\%) as non-amplified. Of those dual tested, 373/411 (90.8\%) were equivocal (2+) by immunohistochemistry, with the corresponding fluorescence in situ hybridization identifying 65/373 (17.4\%) as HER2-amplified, 27/ $373(7.2 \%)$ as equivocal, and $281 / 373(75.3 \%)$ as non-amplified. Dual testing identified 18/411 (4.4\%) as positive (3+) by immunohistochemistry, with the corresponding fluorescence in situ hybridization identifying $15 / 18(83.3 \%)$ as amplified and 3/18 $(16.7 \%)$ as non-amplified. When the immunohistochemistry score was negative (0 or $1+$ ), the rate of HER2 amplification identified in the micropapillary morphology group with the FDA-approved probe set was $55 \%$ (5/9 with ratios ranging from 2.1 to 2.3 and average HER2 copy numbers ranging from 4.1 to 7.2 ).

In the current series, the majority of cases included were received for testing in a reference laboratory setting. As such, we were not able to obtain treatment or outcome data for these patients. Press et al have recently published a retrospective assessment of HER2 fluorescence in situ hybridization ASCO/CAP 2013 guidelines as applied to the Breast Cancer International Research Group (BCIRG) -005, BCIRG-006, and BCIRG-007 clinical trials, which are large cohorts with long-term clinical follow-up. ${ }^{18}$ The 2013 guidelines using dual probes identify three groups as ISH-amplified (group 1 with a HER2/ Chromsome 17 centromere ratio of $\geq 2.0$ and an average HER2 copy number of $\geq 4.0$; group 2 has a ratio $\geq 2.0$ and an average HER2 copy number of $<4$; group 3 has a ratio $<2.0$ and an average HER2 copy number of $\geq 6.0$ ). Correlating fluorescence in situ hybridization results with outcome, the authors make the point that patients in group 1 benefitted from trastuzumab in BCIRG-006, whereas those in 

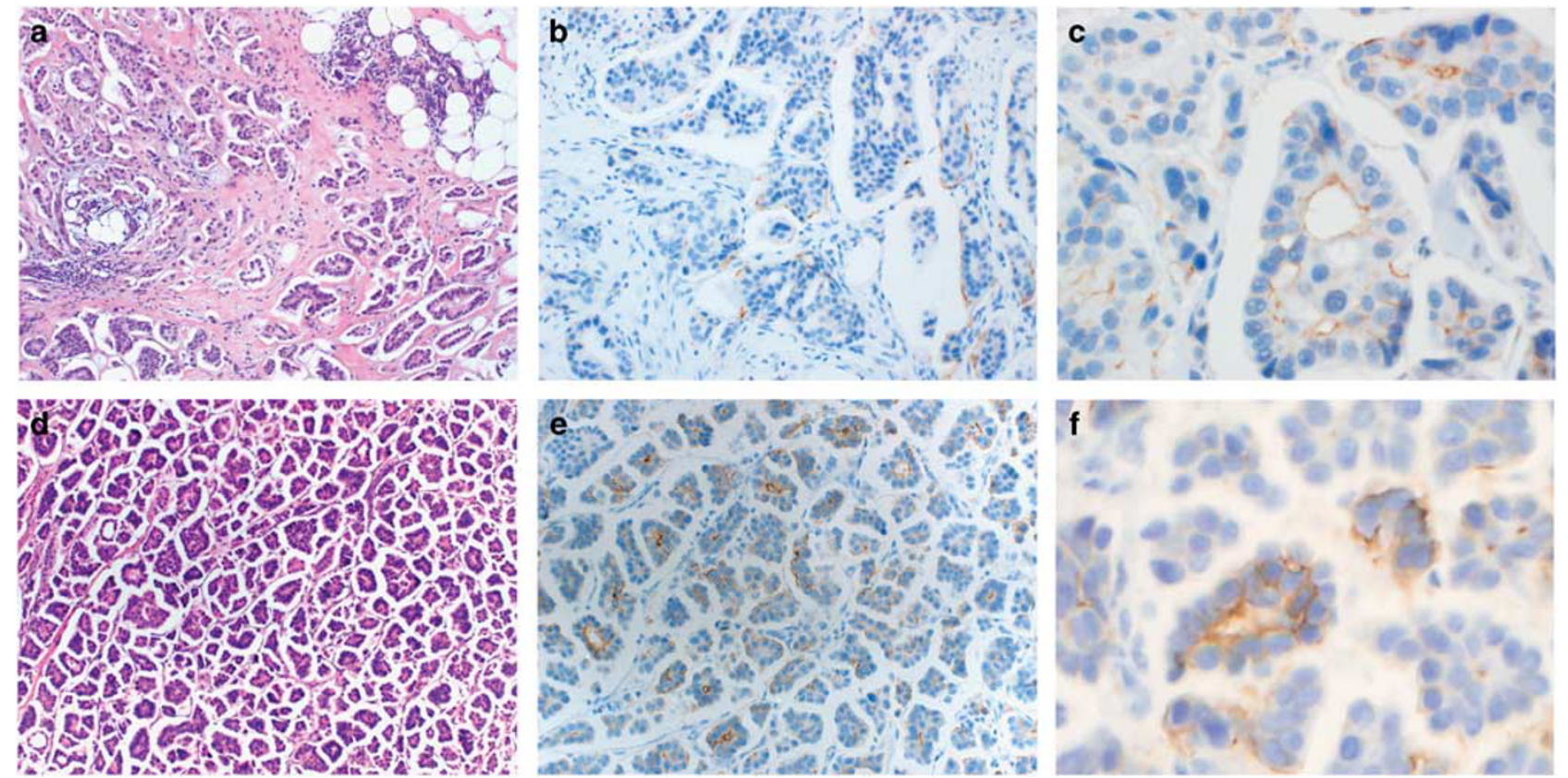

Figure 1 Examples of invasive carcinomas with micropapillary features (a and $\mathbf{d}$ ) and corresponding HER2 immunohistochemistry stains at $\times 20$ (b and $\mathbf{e}$ ) and $\times 40$ (c and $\mathbf{f}$ ) that were scored as 1+, yet were HER2-amplified by fluorescence in situ hybridization. Note weak staining in the apical lumens and focal weak lateral immunoreactivity.

group 2 did not show a benefit from HER2-targeted therapy. Drawing from the data from Press et al, one would expect that the five cases identified as fluorescence in situ hybridization amplified by the FDA-approved probe set and falling into group 1 (ratio $\geq 2.0$ and HER2 copy number $>4.0$ ) would derive benefit from HER2-targeted therapy. In our series, two of the fluorescence in situ hybridizationamplified cases fell into group 3 (ratio $<2$ and HER2 copy number $\geq 6.0$ ). Press et al found that only 55 $(0.5 \%)$ of the 10468 breast cancers screened for BCIRG-005, -006, and -007 fell into group 3, and, overall, the authors considered the number insufficient for definitive evaluation of response in BCIRG-005 or -006. In our study, two cases that were fluorescence in situ hybridization equivocal by the FDA-approved probe set (ratio $<2$, average HER2 copy number $\geq 4$ and $<6$ ) were found to be amplified by an alternative chromosome 17 probe (HER2/RAI1 with ratios of 2.1 and 2.2 and HER2 copy numbers of 5.2 and 4.3, respectively). Although the ASCO/CAP 2013 guidelines suggest the possibility of resolving equivocal status with an alternative probe, there are no clinical trials that have systematically evaluated response to HER2-targeted therapy in this group of patients. That said, the equivocal group of patients re-evaluated by Press et al (group 4, ratio $<2$ with HER2 copies $\geq 4$ and $<6$ ) was accrued to BCRIG-005 to a chemotherapy arm without HER2-targeted therapy and had similar outcomes (disease-free and overall survival) to those patients who were non-amplified (group 5, ratio $<2$ and HER2 copies < 4.0).
As anti-HER2 therapy is an important treatment option, we believe that an in situ hybridization testing methodology should be sought in micropapillary carcinomas that are immunohistochemistrynegative, given the discordance described herein. Studies in larger cohorts and evaluation of response to anti-HER2 therapy in patients with micropapillary carcinoma will help to further clarify this issue.

In conclusion, this study supports the ASCO/CAP recommendation that pathologists should consider reporting immunohistochemistry in this morphology as equivocal and perform reflex testing using in situ hybridization.

\section{Note Added in Proof}

In the interim between manuscript acceptance and publication, the ASCO/CAP draft recommendations for the focused update to HER2 testing in breast cancer were made available for public comment. If these draft recommendations are implemented, 4/9 FISH amplified micropapillary carcinomas based on the 2013 guidelines would be reclassified. Two cases, both with a HER2/CEN17 ratio $<2$ and a HER2 copy number between 4-6 (equivocal by 2013 guidelines), were amplified by HER2/RAI1 reflex fluorescence in situ hybridization. According to the draft recommendations, alternative probes will no longer be employed and such cases would reflex back to immunohistochemistry and be called negative due to lack of circumferential staining. Two cases, both with HER2/CEN17 ratio $<2$ with average HER2 copy numbers $>6$ are considered amplified 
by the 2013 ASCO/CAP guidelines. According to the draft recommendations, such cases would reflex to immunohistochemistry which would be interpreted as negative due to a lack of circumferential staining.

\section{Disclosure/conflict of interest}

The authors declare no conflict of interest.

\section{References}

1 Wolff AC, Hammond ME, Hicks DG, et al. Recommendations for human epidermal growth factor receptor 2 testing in breast cancer: American Society of Clinical Oncology/College of American Pathologists clinical practice guideline update. J Clin Oncol 2013;31: 3997-4013.

2 Vingiani A, Maisonneuve P, Dell'orto P, et al. The clinical relevance of micropapillary carcinoma of the breast: a case-control study. Histopathology 2013;63:217-224.

3 Willmore-Payne C, Damjanovich-Colmenares K, Pasi $\mathrm{AV}$, et al. Inconsistent results with different secondary reflex assays for resolving HER2 status. Am J Clin Pathol 2016;146:618-626.

4 Wolff AC, Hammond ME, Hicks DG, et al. Reply to E.A. Rakha et al. J Clin Oncol 2015;33:1302-1304.

5 Yang W, Wei B, Chen M, et al. Evaluation of immunohistochemistry HER2 results interpretation in invasive micropapillary carcinoma of the breast. Zhonghua Bing Li Xue Za Zhi 2015;44:48-52.

6 Abrahao-Machado LF, Scapulatempo-Neto C. HER2 testing in gastric cancer: an update. World J Gastroenterol 2016;22:4619-4625.

7 Bang YJ, Van Cutsem E, Feyereislova A, et al. Trastuzumab in combination with chemotherapy versus chemotherapy alone for treatment of HER2-positive advanced gastric or gastro-oesophageal junction cancer (ToGA): a phase 3, open-label, randomised controlled trial. Lancet 2010;376:687-697.

8 Gruel N, Benhamo V, Bhalshankar J, et al. Polarity gene alterations in pure invasive micropapillary carcinomas of the breast. Breast Cancer Res 2014;16:R46.
9 Gruel N, Fuhrmann L, Lodillinsky C, et al. LIN7A is a major determinant of cell-polarity defects in breast carcinomas. Breast Cancer Res 2016;18:23.

10 Shelly M, Mosesson Y, Citri A, et al. Polar expression of ErbB-2/HER2 in epithelia. Bimodal regulation by Lin-7. Dev Cell 2003;5:475-486.

11 Marchio C, Iravani M, Natrajan R, et al. Genomic and immunophenotypical characterization of pure micropapillary carcinomas of the breast. J Pathol 2008;215: 398-410.

12 Cancer Genome Atlas N. Comprehensive molecular portraits of human breast tumours. Nature 2012;490: 61-70.

13 Gokce H, Durak MG, Akin MM, et al. Invasive micropapillary carcinoma of the breast: a clinicopathologic study of 103 cases of an unusual and highly aggressive variant of breast carcinoma. Breast J 2013;19: 374-381.

14 Badyal RK, Bal A, Das A, et al. Invasive micropapillary carcinoma of the breast: immunophenotypic analysis and role of cell adhesion molecules (CD44 and E-cadherin) in nodal metastasis. Appl Immunohistochem Mol Morphol 2016;24: 151-158.

15 Varga Z, Zhao J, Ohlschlegel C, et al. Preferential HER-2/neu overexpression and/or amplification in aggressive histological subtypes of invasive breast cancer. Histopathology 2004;44:332-338.

16 Kuroda H, Sakamoto G, Ohnisi K, et al. Overexpression of Her2/neu, estrogen and progesterone receptors in invasive micropapillary carcinoma of the breast. Breast Cancer 2004;11:301-306.

17 Pettinato G, Manivel CJ, Panico L, et al. Invasive micropapillary carcinoma of the breast: clinicopathologic study of 62 cases of a poorly recognized variant with highly aggressive behavior. Am J Clin Pathol 2004;121:857-866.

18 Press MF, Sauter G, Buyse M, et al. HER2 gene amplification testing by fluorescent in situ hybridization (FISH): comparison of the ASCOCollege of American Pathologists Guidelines With FISH Scores Used for Enrollment in Breast Cancer International Research Group Clinical Trials. J Clin Oncol 2016; pii: JCO666693 (e-pub ahead of print, 29 August 2016). 